\title{
Ecological and Geographical Separation of Three Varieties of Sporobolus vaginiflorus (Poaceae) in Eastern Ontario
}

\author{
PAUl M. CATLing
}

Agriculture and Agri-Food Canada, Environmental Health, Biodiversity, Saunders Building, Central Experimental Farm, Ottawa, Ontario K1A 0C6 Canada; email: catlingp@agr.gc.ca

Catling, Paul M. 2013. Ecological and geographical separation of three varieties of Sporobolus vaginiflorus (Poaceae) in eastern Ontario. Canadian Field-Naturalist 127(2): 155-163.

Field and herbarium studies of the three varieties of Sporobolus vaginiflorus (var. inaequalis, var. vaginiflorus, and var. ozarkanus) in eastern Ontario found that (1) there is a strong tendency for the varieties to occur alone; (2) var. inaequalis occurs primarily on roadside gravels, river shores, and granite barrens and is the only variety associated with acid as well as alkaline substrates; and (3) both var. vaginiflorus and var. ozarkanus are confined to alvar landscapes but occupy different geographical regions. The ecological and geographical differentiation of these taxa supports their taxonomic recognition and the protection of alvar landscapes to conserve subspecific variants.

Key Words: Sporobolus vaginiflorus; Sporobolus vaginiflorus var. vaginiflorus; Sporobolus vaginiflorus var. inaequalis; Sporobolus vaginiflorus var. ozarkanus; Poaceae; ecology; distribution; alvar; granite barren; taxonomy; classification; identification; Ontario

The three annual varieties of Sporobolus vaginiflorus (Torr. ex A. Gray) Alph. Wood (Sheathed Dropseed) that occur in eastern Ontario and throughout much of eastern North America include var. inaequalis Fernald, var. ozarkanus (Fernald) Shinners, and var. vaginiflorus. These varieties were accepted by Fernald $(1933,1950)$ and Dore and McNeill (1980). Dore and McNeill (1980) reported distributional differences but did not relate them to ecology. There has been no further study of distributional differences, and more recent authors have generally accepted two taxa (vars. vaginiflorus and ozarkanus), placing var. inaequalis as a synonym of var. vaginiflorus (Peterson et al. 2003).

The regional study reported here aims to provide a better understanding of the ecology and distribution of the varieties of Sporobolus vaginiflorus in eastern Ontario and a more informed classification.

\section{Methods}

Three procedures were employed to gather information on the ecology and distribution of the three taxa.

\section{(1) Co-occurrence data}

Two hundred individual plants at each of the 49 sites (up to 1 acre each $\left(4046.86 \mathrm{~m}^{2}\right)$, including 4 river shores, 23 alvars (areas of flat, naturally more or less open limestone), and 22 roadsides), were studied and identified in the field in August to November 2009 in order to provide information on co-occurrence. Representative voucher specimens for each taxon were collected from most sites and deposited at the National Collection of Vascular Plants, Agriculture and Agri-Food Canada, Ottawa (DAO, acronyms follow Thiers 2013).

\section{(2) Habitat information from specimens}

All specimens of Sporobolus vaginiflorus from eastern Ontario in the herbaria at the National Herbarium of
Canada, Canadian Museum of Nature, Ottawa (CAN), at the National Collection of Agriculture and Agr-Food Canada, Ottawa (DAO), and at the Fowler Herbarium, Biology Department, Queen's University, Kingston (QK), were identified and associated with river shore, alvar, roadside, and granite habitats based on available specimen label data, in order to characterize the habitats of the varieties using frequency histograms.

\section{(3) Geographic distribution}

All herbarium specimens of the three varieties were plotted on maps of eastern Ontario showing alvar landscapes to provide a visual association and to expand use of the data for specimens lacking habitat information. Alvar landscapes for the latter application were derived from a plot of undulating, 1-3\% slope class landscapes with melanic brunisol soils with calcareous clay or loam parent material (Agriculture Canada 1989). This provided reasonable boundaries for the Smiths Falls (central on the maps) and Napanee (lower left on maps) limestone plains as they have been portrayed previously (Chapman and Putnam 1984). The herbarium data included 184 (Appendix 1) specimens, of which 83 were referable to var. inaequalis, 46 to var. ozarkanus, and 55 to var. vaginiflorus. Plants were identified using a newly developed key to northeastern North American Sporobolus taxa based on Dore and McNeill (1980):

1a. Lemmas smooth, hairless; white, lacking grey mottling but sometimes suffused with purple near their tips if exposed . . . . Sporobolus neglectus

1b. Lemmas with minute antrorse hairs, or without; whitish with grey mottling, except those near the long exposed tips of a terminal inflorescence which may become uniformly dark reddishpurple $\ldots \ldots \ldots \ldots \ldots \ldots \ldots \ldots \ldots \ldots \ldots \ldots \ldots \ldots \ldots \ldots$ 
2a. Paleas of lower ensheathed florets on any inflorescences long, acute, and prolonged beyond the lemma (1.2-2 times as long as the lemma) ..... 3

2b. Paleas of lower ensheathed florets on any inflorescences mucronate, only slightly exceeding the lemma (less than 1.2 times as long as the lemma) ..................... 5

3a. Lemma and palea without antrorse hairs $S$. vaginiflorus var. inaequalis $\times$ ozarkanus $\ldots \ldots \ldots$ ?

3b. Lemma and palea with antrorse hairs ........ 4

\section{Results}

\section{(1) Co-occurrence}

At 42 of 49 sites (85.7\%), only one variety of Sporobolus vaginiflorus was present. There were 5 sites $(10.2 \%)$ with two varieties (in all cases var. inaequalis and var. ozarkanus) and only 2 sites with all three varieties $(4.1 \%)$. There is a clear tendency for varieties to occur alone based on examination of a total of 9800 individuals at 49 sites (Appendix 1, Table 1).

\section{(2) Habitat information from specimens}

Of the three taxa, Sporobolus vaginiflorus var. inaequalis has the broadest ecological amplitude based on specimen label data (Figure 1), but it occurs primarily along roads. The granite and sand habitats are often acidic but roadsides and other habitats may have neutral or alkaline substrates. In eastern Ontario, var. ozarkanus is confined to natural habitats of alvars, and var. 4a. Paleas of ensheathed florets long, narrowly acute, or acuminate and 1.2-2 times as long as the lemma ........... vaginiflorus var. inaequalis

4b. Paleas of ensheathed florets acute or rounded and 1.1-1.2 times as long as the lemma?

$\ldots \ldots \ldots \ldots \ldots$. vaginiflorus var. inaequalis $\times$ var. vaginiflorus

5a. Lemmas strigose with antrorse hairs (shiny microhairs visible in directed light) . . . . . S vaginiflorus var. vaginiflorus

\section{5b. Lemmas not strigose}

.............. vaginiflorus var. ozarkanus

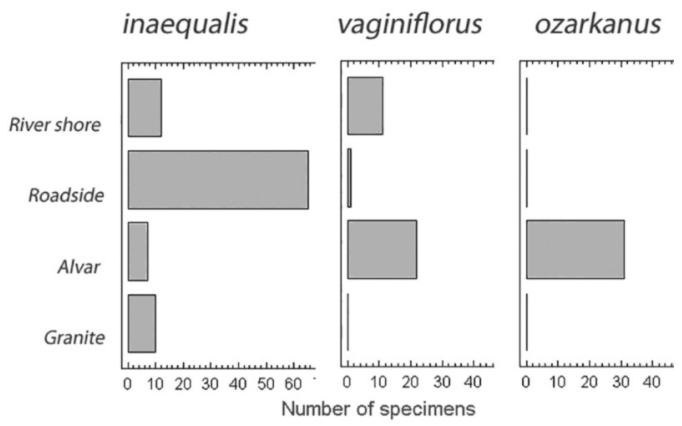

FIGURE 1. Histograms showing the number of plants of the three different varieties of Sporobolus vaginiflous (var. inaequalis, var. vaginiflorus, and var. ozarkanus) in different habitats based on specimens at CAN, DAO, and QK.

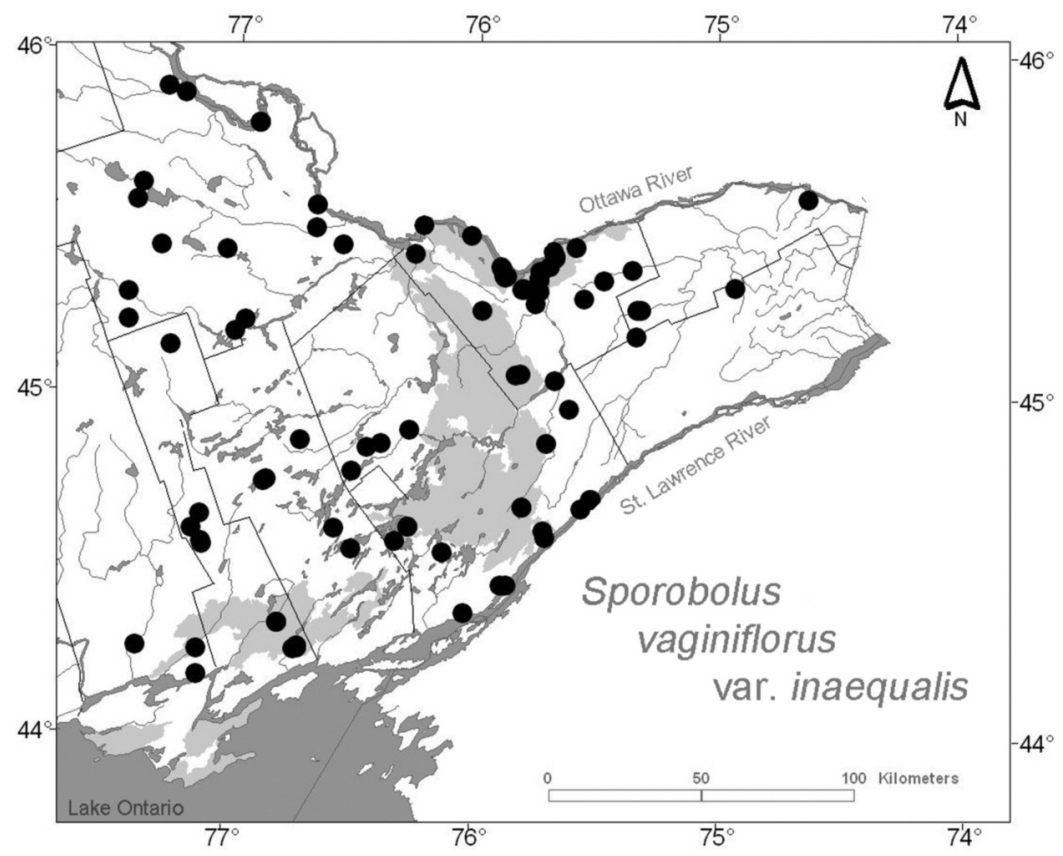

FIGURE 2. Collection sites of Sporobolus vaginiflorus var. inaequalis in eastern Ontario based on specimens examined at CAN, $\mathrm{DAO}$, and QK. The alvar landscapes are lightly shaded, and the map portrays primary drainage and county boundaries. 


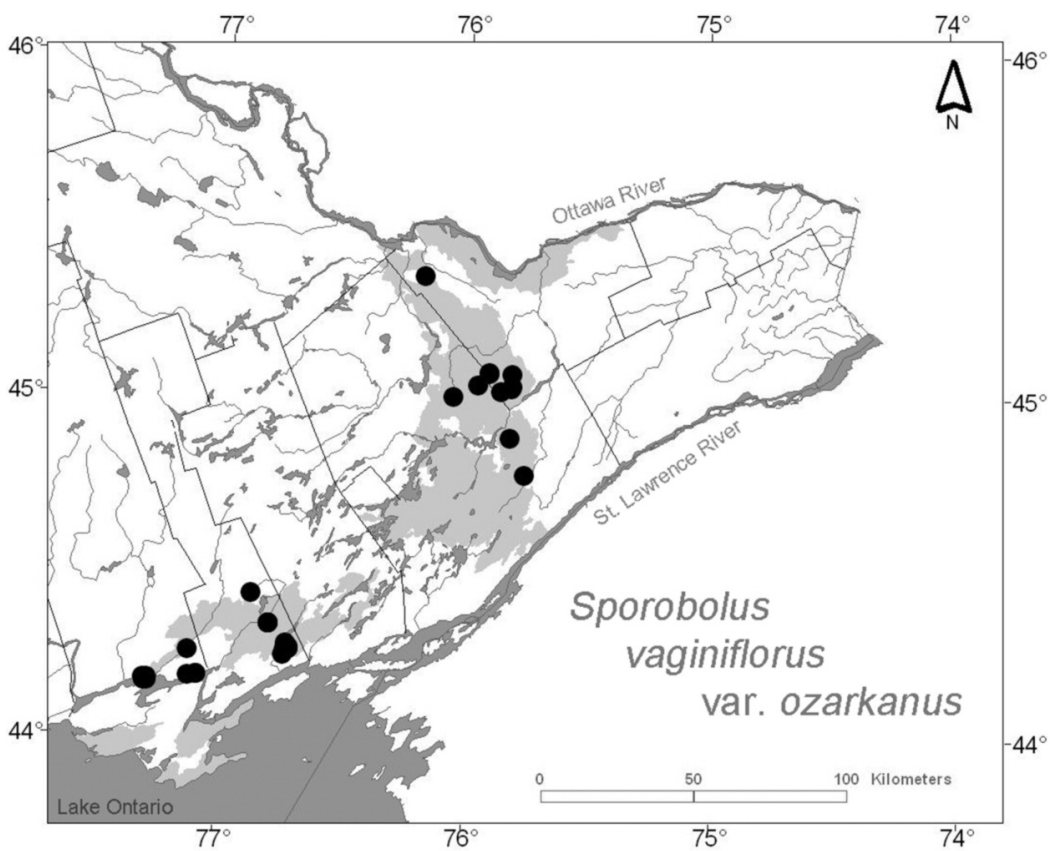

FIGURE 3. Collection sites of Sporobolus vaginiflorus var. ozarkanus in eastern Ontario based on specimens examined at CAN, $\mathrm{DAO}$, and QK. The alvar landscapes are lightly shaded, and the map portrays primary drainage and county boundaries.

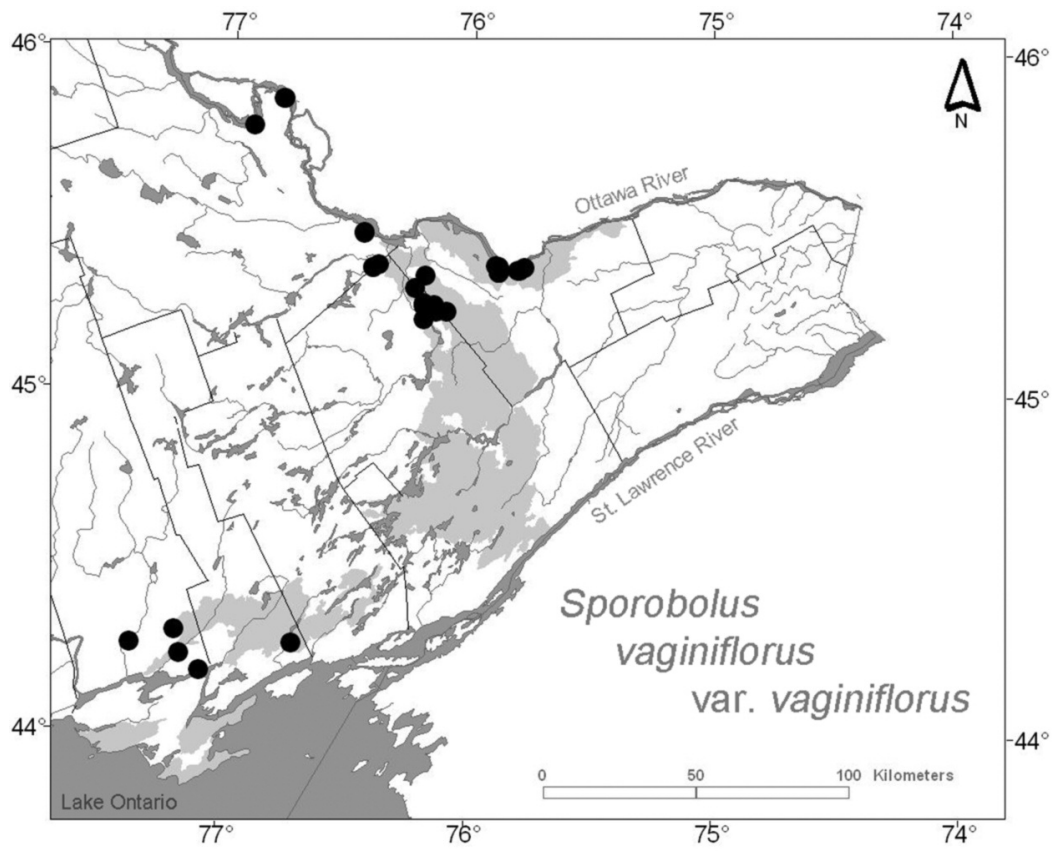

FIGURE 4. Collection sites of Sporobolus vaginiflorus var. vaginiflorus in eastern Ontario based on specimens examined at $\mathrm{CAN}, \mathrm{DAO}$, and QK. The alvar landscapes are lightly shaded, and the map portrays primary drainage and county boundaries. 
vaginiflorus occurs mostly in alvars and on river shores (Figure 1). Some plants attributed to var. vaginiflorus from river shore habitats, such as Shirleys Bay on the Ottawa River, are intermediate between var. inaequalis and var. vaginiflorus.

\section{(3) Geographic distribution}

The widespread occurrence of Sporobolus vaginiflorus var. inaequalis (Figure 2) corresponds to the map produced by Dore and McNeill (1980, map 164). Both var. vaginiflorus and var. ozarkanus have a more extensive distribution in eastern Ontario than was shown by Dore and McNeill (1980), but the seminal observation by Dore and McNeill (1980) — that the distribution was restricted - is supported (Figures 3 and 4). The distinctive geographic distributions of these two varieties in eastern Ontario are associated with alvar landscapes, but the two varieties occur largely in different subunits of those landscapes, var. vaginiflorus occurring in the Napanee and northern Smiths Falls plains and var. ozarkanus occurring in the Napanee and southern Smiths Falls plains. The much more extensive distribution of var. inaequalis is not surprising, considering the variety of habitats that it occupies.

\section{Discussion}

The strong tendency for varieties of Sporobolus vaginiflorus to occur alone was anticipated, since many herbarium sheets have numerous individual plants and yet more than one taxon was mounted on a sheet less than $1 \%$ of the time. To a large extent, the tendency to occur alone may be due to the demonstrated differences in ecology. Varieties and subspecies may often be ecologically different, as species are (Van Valen 1976; Grant 1992), with some exceptions (Shmida and Ellner 1984; Scheffer and van Nes 2006). As a consequence, ecological differentiation may be useful in supporting the recognition of infrataxa. Since the three varieties are ecologically and morphologically distinct, they are more than likely genetically unique. Observations elsewhere in the range of $S$. vaginiflorus are needed to confirm that recognition of the varieties is warranted.

\section{Acknowledgements}

Assistance in gathering data was provided by Brenda Kostiuk and Gisèle Mitrow.

\section{Literature Cited}

Agriculture Canada. 1989. Soil landscapes of Canada, Ontario-South. Canada Soil Inventory, Land Resource Centre, Research Branch, Agriculture Canada, Contribution Number 87-17. 8 pages + attribute. http://sis.agr.gc.ca/cansis /nsdb/slc/v3.1.1/index.html.

Chapman, L. J., and D. F. Putnam. 1984. The physiography of southern Ontario. University of Toronto Press, Toronto, Ontario. 270 pages + map P.2715, Physiography of southern Ontario, (1:6000 000).

Dore, W. G., and J. McNeill. 1980. Grasses of Ontario. Monograph 26, Research Branch, Agriculture Canada, Ottawa, Ontario.

Fernald, M. L. 1933. Two segregates in Sporobolus. Rhodora 35: $108-110$.

Fernald, M. L. 1950. Gray's manual of Botany. Eighth edition. American Book Company, New York, New York. 1632 pages.

Grant, V. 1992. Comments on the ecological species concept. Taxon 41(2): 310-312.

Peterson, P. M., S. L. Hatch, and A. S. Weakley. 2003. 17.30 Sporobolus R. Br. Pages 115-139 in Magnoliophyta: Commelinidae (in part): Poaceae, part 2. Edited by M. E. Barkworth, K. M. Capels, S. Long, and M. B. Piep. Vol. 25 of the Flora of North America North of Mexico. Edited by the Flora of North America Editorial Committee. Oxford University Press, New York, New York.

Scheffer, M., and E. H. van Nes. 2006. Self-organized similarity, the evolutionary emergence of groups of similar species. Proceedings of the National Academy of Sciences 103: 6230-6235.

Shmida, A., and S. Ellner. 1984. Coexistence of plant species with similar niches. Vegetatio 58: 29-55.

Thiers, B. 2013. Index Herbariorum: A global directory of public herbaria and associated staff. New York Botanical Garden Virtual Herbarium. http://sciweb.nybg.org/science2 /IndexHerbariorum.asp.

Van Valen, L. 1976. Ecological species, multispecies, and oaks. Taxon 25: 233-239.

Received 13 March 2013

Accepted 17 April 2013 
APPENDIX 1. Specimens examined that are the basis for the mapping of the varieties of Sporobolus vaginiflorus in eastern Ontario. Under each variety, the herbarium sheet citations are organized by collection date. Sheets lacking dates are listed at the end. An additional list of site records by the author (not vouchered) on file at DAO (also mapped) is included. The latitudes and longitudes are generally accurate to within $0.5 \mathrm{~km}$ of collection sites for collections after 1970 but may be up to $5 \mathrm{~km}$ distant from collection sites prior to that date, when it was more common to give general locations. The approximate latitude and longitude were added to the label data later for mapping purposes.

\section{Sporobolus vaginiflorus var. inaequalis}

Specimens. CANADA. Ontario: Merivale, 45.3568, -75.7369, 30 September 1935, W.G. Dore 223 (DAO 221100); Merivale, 45.3568, -75.7369, 30 September 1935, W.G. Dore 224 (DAO 221101); Merivale, 45.3568, -75.7369, 18 September 1936, W.G. Dore 405 (DAO 221102); Bells Corners, 45.3291, -75.8055, 8 October 1936, W.G. Dore 406 (DAO 221099); Galetta, 45.4256, -76.256, 3 September 1943, W.G. Dore \& H. Groh 5069 (DAO 221042); Ennis Point, 45.3864, -75.8938, 3 September 1948, W.G. Dore \& A.J. Breitung 9277 (DAO 221097); Innis Point, 45.3864, -75.8938, 3 September 1948, W.G. Dore \& A.J. Breitung 9298 (DAO 221098); Sand Point [3 km SSE of Westmeath] 45.8000, -76.9166, 19 September 1948, W.G. Dore 9765 (DAO 221095); Shirleys Bay, 45.3658, -75.8828, 7 September 1952, W.G. Dore 14035 (DAO 221047); Fermoy, 44.6159, -76.5719, 2 October 1952, W.G. Dore \& J.M. Gillett 17569 b (DAO 221089); Westport, 44.6852, -75.7984, 2 October 1959, W.G. Dore \& J.M. Gillett 43870 (DAO 596334); 8 miles of Eganville, 45.4279, -77.0403, 22 August 1960, W.G. Dore 18344 (DAO 221090); Chaffeys Road, 44.622, -76.2667, 10 September 1960, $R$. Hainault 1335 (DAO 221091); Chaffeys Road, 44.622, -76.2667, 10 September 1960, R. Hainault 1335 (DAO 839503); S. Crosby Twp., road to Chaffeys 1 mile from Rd. 15, 44.5808, -76.3172, 10 September 1960, R. Hainault 1335 (QK 49047); Prescott (3 miles NE of centre), 44.7095 , -75.5138, 14 September 1960, W.G. Dore 18582 (DAO 221076); Prescott (3 miles NE of centre), 44.7119, -75.5169, 14 September 1960, W.G. Dore 18582 (QK 49046); Prescott, 44.7095, -75.5138, 24 September 1960, W.G. Dore 18703 (DAO 221075); Prescott (3.2 miles SW), Hwy. 2 along river shore, 44.6814, -75.5559, 24 September 1960, W.G. Dore 18703 (QK 49045); Mallorytown, 44.4544, -75.8805, 5 September1961, W.G. Dore 19603 (DAO 221072); Mallorytown Landing, 44.4544, -75.8591, 6 September 1961, W.G. Dore 19657 (DAO 221073); N of Brockville, 44.6178, -75.7106, 6 September1962, W.G. Dore 20065 (DAO 221088); Petawawa (one mile WNW), 45.9015, -77.3057, 21 July 1965, W.G. Dore \& T.C. Brayshaw s.n. (CAN 569754); Rear of Leeds \& Lansdowne Twp., $3 \mathrm{~km}$ NE of Lyndhurst, 44.5497, -76.1231, 30 September 1966, Beschel 15758 (QK 62927); Bedford Twp., Frontenac Park, Little Clear Lake, 44.55, -76.5000, 1 September 1968, R. Hainault \& I. Macdonald 4718 (QK 97376); Camden East alvar, 44.3342, -76.7929, 26 August 1969, Hainault \& MacDonald 5329 (DAO 839507); Camden Twp., Camden East alvar, NE sector, 44.3342, -76.7929, 26 August 1969, R. Hainault \& I. Macdonald 5329 (QK 90839); Highway to Barry's Bay and S exit to Killaloe, 45.5654, -77.4205, 27 September 1970, M.I. Moore 5285 (CAN 569753); Belleville, Thurlow Twp., Canadian Pacific Railway (CPR) abandoned cinder track, 44.2567, -77.3697, 6 October 1970, S. Vander Kloet 90 (QK 97721); August-October North Algoma Twp., N of Golden Lake, Thomas Mountain, 45.6167, -77.4000, 14 September
1978, J.M. Gillett \& M.J. Shchepanek 17988 (CAN 417855); Bishops Mills, 44.8725, -75.7015, 28 August 1979, W.G. Dore 493 (DAO 689350); Nepean, Barrhaven, $1 \mathrm{~km} \mathrm{SW}$ of intersection of Fallowfield and Greenbank Rds., 45.2833, -75.75000, 16 September 1979, D.F. Brunton, J.D. Lafontaine \& C. Frankton 1980 (CAN 490172); Ottawa, 1030 Derby Street, 45.3786, -75.7322, 17 September 1979, D.F. Brunton 1981 (CAN 452556); September, August, August Nepean, 45.3833, -78.9500, 11 September 1980, D.F. Brunton 2730 (DAO 282956); Point Pelee National Park parking lot, by marsh boardwalk, 41.9678, -82.5308, 17 October 1981, D.F. Brunton 3509 (CAN 479997); Petawawa, Petawawa Fish Hatchery, Laurentian Drive, 45.8833, -77.2333, 28 July 1982, D.F. Brunton 3653 (CAN 470169); Front of Leeds \& Lansdowne Twp., Fitzsimmons Mountain, 44.3722, -76.0333, 4 September 1982, S.J. Darbyshire, D.A. Sutherland, \& M. Oldham 1738 (CAN 468804); Gloucester, the Queensway (Hwy. 417), SE side of Montreal Road interchange, 45.4500, -75.5833, 9 September 1982, D.F. Brunton 3975 (CAN 470411); E of Marionville, 45.1902, -75.3306, 15 June 1983, S.J. Darbyshire \& C. Hanrahan 1878 (DAO 353135); Bell Corners, 45.3291, -75.8055, 29 August 1983, S.G. Aiken s.n. (DAO 398739); Maxville exit on hwy 417, 45.3347, -74.9207, 31 August 1983, S.G. Aiken \& S.J. Darbyshire 2601 (DAO 376329); $3 \mathrm{~km} \mathrm{~W}$ of Embrun, 45.2683, -75.329, 3 September 1983, S.J. Darbyshire 2091 (DAO 396191); Russell Twp., $3 \mathrm{~km}$ west of Embrun north of Castor River, 45.2694, -75.3125, 3 September 1983, S.J. Darbyshire 2091 (CAN 531276); Horton Twp., $3 \mathrm{~km} \mathrm{SW}$ of Chenaux, 45.5625, -76.6666, 5 September 1983, S.J. Darbyshire 2112 (CAN 531275); Rideau River Provincial Park north of Kemptville, 45.0583, -75.6694, 11 September 1983, S.J. Darbyshire 2117 (CAN 531272); Ottawa, NCC driveway along Rideau Canal [Queen Elizabeth Drive] near Pretoria bridge, 45.4372, -75.679, 6 September 1984, S.J. Darbyshire (CAN 531283); Howard Twp., Hwy 401 at turn-off for Ridgetown, 42.4833, -81.9166, 20 September 1984, S.J. Darbyshire 2443 (CAN 531286); Ottawa, NCC driveway [Queen Elizabeth Drive], 45.3943, -75.6957, 22 September 1984, W.G. Dore 936 (DAO 452166); Hwy. 16 and McGovern Road, SE of Kemptville, 44.9745, -75.6069, 14 June 1985, W.G. Dore 973 (DAO 444402); Westmeath, 45.8000, -76.9166, 22 September 1985, S.J. Darbyshire, A.A. \& S.A. Reznicek, P.M. Catling \& V. Brownell 2887 (DAO 551870); Westmeath Twp., Ottawa River, Sand Point, 45.8000, -76.9166, 22 September 1985, S.J. Darbyshire, A.A. \& S.A. Reznicek, P.M. Catling \& V. Brownell 2887 (CAN 500148); Bruce pit, 45.3249, -75.7985, 6 October 1985, S.J. Darbyshire and S.G. Aiken 2896 (DAO 551871); Nepean, Bells Corners, Bruce pit, 45.325, -75.8083, 6 October 1985, S.J. Darbyshire \& S.G. Aiken 2896 (CAN 531281); NW of Mountain, 44.7500, -76.8666, 21 August 1986, S.G. Darbyshire \& C. Hanrahan 3182 (DAO 465544); Little Clear Lake, 44.555, -76.4996, 1 September 1986, Hainault \& MacDonald 4718 (DAO 839506); Rideau Twp., $13 \mathrm{~km} \mathrm{~S} \mathrm{of}$ 
Richmond, Cedar Grove Picnic Site at edge of corn field, 45.0722, -75.8306, 14 September 1986, S.J. Darbyshire \& V.P. Shepstone 3202 (CAN 520897); Gloucester Twp., on Hawthorne Road extension, 45.3000, -75.55, 1 October 1986, A.W. Dugal \& M. Camfield 2282 (CAN 522505); Clarendon, 44.8714, -76.7158, 30 August 1987, S.J. Darbyshire 3606 (DAO 575637); Oso Twp., $1.5 \mathrm{~km}$ west of Clarendon Station, 44.8666, -76.3833, 30 August 1987, S.J. Darbyshire 3606 (CAN 529764); Asselstine alvar, 44.2583, -76.725, August 1988, P.M. Catling s.n. (DAO 580227); South Sherbrooke Twp., $3 \mathrm{~km}$ west of Christie Lake, $1 \mathrm{~km} \mathrm{~N}$ of Tay River, near Canadian Pacific Railway (CPR) trestle, 44.7833, -76.5000, 21 August 1989, M.J. Shchepanek, A.W. Dugal, R.R. Ireland \& L.M. Ley 8521 (CAN 542960); Ottawa River near Baskin's Beach, 45.4823, -76.0209, 4 October 1991, J.M. Gillett 18058 (CAN 562960); Kaladar Jack Pine Area of Natural and Scientific Interest (ANSI), 44.607, -77.157, 26 September 1993, V.R. Brownell s.n. (DAO 671186); Morris Island Conservation Area, Ottawa River, 45.4500, -76.2833, 30 August 1997, W.J. Crins, H.M. Runtz \& D. Runtz 10589 (DAO 865841); Canadian Forces Base (CFB) Petawawa, $1 \mathrm{~km}$ NE of Montgomery Lake, $100 \mathrm{~m} \mathrm{~W}$ of tower at top of Montgomery Tower Road, 46.9416, -77.55, 25 September 1998, D.F. Brunton 13810 (CAN 585313); Mellon Lake [9.5 km S of Kaladar], 44.5596, -77.1116, 31 August 2004, S.J. Darbyshire and C. Hanrahan 5252 (DAO 797766); Constance Bay sand hills, Bishop Davis Road and Allbirch Road, 45.4916, -75.0875, 9 October 2007, S.J. Darbyshire \& C.V. Hanrahan 5595 (DAO 877548); Loyalist Twp., Odessa, west of Bridge St., 44.2663, -76.7104, 26 August 2009, D. Kristensen (QK 139024); Odessa alvar, 44.2663, -76.7104, 18 October 2009, P.M. Catling \& B. Kostiuk s.n. (DAO 844864); Deseronto Airport, 44.1776, -77.1195, 18 October 2009, P.M. Catling \& B. Kostiuk s.n. (DAO 845581); Camden East, 44.3342, -76.7929, 18 October 2009, P.M. Catling \& B. Kostiuk s.n. (DAO 845605); Camden East, 44.3342, -76.7929, 18 October 2009, P.M. Catling \& B. Kostiuk s.n. (DAO 845661); Camden East, 44.3342, -76.7929, 18 October 2009, P.M. Catling \& B. Kostiuk s.n. (DAO 845608); Lonsdale, 44.2531, -77.1221, 18 October 2009, P.M. Catling \& B. Kostiuk s.n. (DAO 845599); sandy edge of Slack Road, Ottawa, 45.3198, -75.7357, 6 November 2009, P.M. Catling s.n. (DAO 845565); Marlborough Forest, 45.0775, -75.8118, 7 November 2009, P.M. Catling s.n. (DAO 845559); Hwy. 417 W of Boundary Road, 45.3548, -75.4678, 8 November 2009, P.M. Catling s.n. (DAO 845566); Shirleys Bay at foot of Rifle Road, 45.3658, -75.8828, 10 November 2009, P.M. Catling s.n. (DAO 845568); Hwy. $41 \mathrm{~N}$ of Maguire, 44.5688, -77.1165, 11 November 2009, P.M. Catling s.n. (DAO 845576); Innis Point near tip, 45.3864, -75.8938, 15 November 2009, P.M. Catling \&B. Kostiuk s.n. (DAO 845571); Innis Point N side, 45.3915, -75.8981, 15 November 2009, P.M. Catling \& B. Kostiuk s.n. (DAO 845573); Westport, 44.6852, -75.7984, s.d., W.G. Dore \& J.M. Gillett s.n. (DAO 221092); Sandy Hill, Ottawa, 45.4252, -75.6735, s.d., I. Zgierska s.n. (DAO 460483).

Sight records. CANADA. Ontario: Hwy. 417 near Vars, 45.386, -75.3486, 8 November 2009, P.M. Catling; Hwy. 417 E of Casselman, 45.364, -75.8726, 8 2009, P.M. Catling; Hwy. 17 at Hawkesbury, 45.5951, -74.6157, 8 2009, P.M.
Catling; Merivale Rd. median S of Baseline Rd. near Campiano Drive, 45.3568, -75.7369, 8 November 2009, P.M. Catling; Hwy. $7 \mathrm{~W}$ of Ottawa, 45.2605, -75.9717, 22 November 2009, P.M. Catling; 12 November 2009, P.M. Catling; Hwy. 7 near Brooke, 44.8544, -76.4431, 12 November 2009, P.M. Catling; Hwy. 7 at Swamp Road, 44.7544, -76.8527, 12 November 2009, P.M. Catling; Kaladar, 44.6482, -77.1217, 12 November 2009, P.M. Catling; W of Arnprior, 45.4468, -76.5566, 22 November 2009, P.M. Catling; Centennial Road at Black Donald Lake, 45.223, -76.9574, 22 November 2009, P.M. Catling; Centennial Road E of LeClaire Lane, 45.1877, -76.9997, 22 November 2009, P.M. Catling; Hwy. 28 at Denbigh, 45.1429, -77.2663, 22 November 2009, P.M. Catling; Hardwood Lake Junction [km W of Renfrew], 45.2129, -77.4401, 22 November 2009, P.M. Catling; Hwy. 515 near Latchford Bridge, 45.2951, -77.4473, 22 November 2009, P.M. Catling; Hwy. 51225 km WSW of Eganville, 45.4339, -77.3162, 22 November 2009, P.M. Catling; Hwy. 417 near Renfrew, 45.4962, -76.6700, 22 November 2009, P.M. Catling; Hwy. 7 near Perth, 44.9091, -76.266,

\section{Sporobolus vaginiflorus var. ozarkanus}

CANADA. Ontario: $1.2 \mathrm{~km} \mathrm{SW}$ of Odessa, 44.2613, -76.7263, 7 September1917, R.W. Hainault 67-315 (DAO 220897); $1 \mathrm{~km} \mathrm{~N}$ of Asselstine, 44.2612, -76.7104, 17 November 1962, R. Beschel et al. 13632 (DAO 221124); Enestown Twp., $3 \mathrm{~km} \mathrm{~S}$ of Odessa near Asselstine, 44.2444, -76.7314, 17 November 1962, Beschel et al. 13632 (QK 54483-1); Hungerford Twp., N end of Dry Lake, 44.4215, -76.8684, 14 September 1968, R. Beschel 17040 (QK 80595); Ernestown Twp., 2.5 miles S of Odessa, 44.2769, -76.7225, 19 September 1968, K.J. Crawford 147 (QK 114247); Camden Twp., Camden East alvar, 44.3342, -76.7929, 4 October 1968, S.P. Vander Kloet 2617 (QK 82077); Rideau Twp., Marlborough Forest, 45.0333, -78.8111, 21 November 1981, S.J. Darbyshire 1602 (CAN 465434); Montagne Twp., lot 5, Conc. X, 45.0444, -75.9500, 16 October 1983, S.J. Darbyshire 2136 (CAN 531277); Ottawa, approx. 0.25 mile E of Hwy. 31, W of Hawthorne Road, 45.3000, -75.33, 23 October 1984, A.W. Dugal 1859 (CAN 501851); Brighton Twp., Presqu'ile Provincial Park, NE side of the main road opposite 2nd Beach Road, 44.0083, -77.7333, 12 September 1986, D.F. Brunton 7024 (CAN 537011); Thurlow Twp., east of Belleville on the Bay of Quinte, Point Anne, 44.1583, -77.2833, 5 September 1987, S.J. Darbyshire, M.J. Oldham, D.A. Sutherland, et al. 3610 (CAN 529768); Thurlow Twp., $4 \mathrm{~km}$ E of Belleville, Point Anne Area of Natural and Scientific Interest (ANSI), 44.1667, -77.2833, 14 September 1990, I.D. Macdonald 21903 (QK 133807); Camden East alvar, 44.3342, -76.7929, 25 August 1993, T. Norris 416 (DAO 685180); Presqu'ile Park, 43.9971, -77.7293, 20 September 1994, S. Blaney (DAO 688881); Camden East alvar, 44.3342, -76.7929, 19 September 1995, M.J. Oldham \& W.D. Bakowsky 18233 (DAO 688980); Mohawk Cemetery, 44.1813, -77.0816, 19 September 1995, M.J. Oldham \& W.D. Bakowsky 18245 (DAO 688983); Point Anne E of Belleville, 44.1575, -77.2949, 20 October 1995, M.J. Oldham \& W.D. Bakowsky 18382 (DAO 701808); Odessa alvar, 44.2663, -76.7104, 18 October 2009, P.M. Catling \& B. Kostiuk s.n. (DAO 844864); Odessa Alvar, 44.2663, -76.7104, 18 October 2009, P.M. Catling \& B. Kostiuk s.n. (DAO 843818); 
Point Anne upper area, W of Belleville, 44.1677, -77.3012, 18 October 2009, P.M. Catling \& B. Kostiuk (DAO 845583); Point Anne lower area, West of Belleville, 44.1575, -77.2949, 18 October 2009, P.M. Catling \& B. Kostiuk (DAO 845533); Deseronto Cemetery, 44.1813, -77.0816, 18 October 2009, P.M. Catling \& B. Kostiuk s.n. (DAO 844865); Deseronto Cemetery, 44.1813, -77.0816, 18 October 2009, P.M. Catling \& B. Kostiuk s.n. (DAO 845531); Deseronto Airport, 44.1776, -77.1195, 18 October 2009, P.M. Catling \& B. Kostiuk s.n. (DAO 845532); Deseronto Airport, 44.1776, -77.1195, 18 October 2009, P.M. Catling \& B. Kostiuk s.n. (DAO 845519); Odessa alvar, 44.2663, -76.7104, 18 October 2009, P.M. Catling \& B. Kostiuk s.n. (DAO 844863); Odessa alvar, 44.2663, -76.7104, 18 October 2009, P.M. Catling \& B. Kostiuk s.n. (DAO 845604); Camden East, 44.3342, -76.7929, 18 October 2009, P.M. Catling \& B. Kostiuk s.n. (DAO 845554); Lonsdale alvar, 44.2531, -77.1221, 18 October 2009, P.M. Catling \& B. Kostiuk s.n. (DAO 845601); Lonsdale alvar, 44.2531, -77.1221, 18 October 2009, P.M. Catling \& B. Kostiuk s.n. (DAO 845660); Lonsdale alvar, 44.2531, -77.1221, 18 October 2009, P.M. Catling \& B. Kostiuk s.n. (DAO 845597); Lonsdale alvar, 44.2531, -77.1221, 18 October 2009, P.M. Catling \& B. Kostiuk s.n. (DAO 845598); Lonsdale alvar, 44.2531, -77.1221, 18 October 2009, P.M. Catling \& B. Kostiuk s.n. (DAO 845602); Camden East, 44.3342, -76.7929, 18 October 2009, P.M. Catling \& B. Kostiuk s.n. (DAO 845610); Camden East, 44.3342, -76.7929, 18 October 2009, P.M. Catling \& B. Kostiuk s.n. (DAO 845556); Marlborough Forest, 45.0775, -75.8118, 7 November 2009, P.M. Catling (DAO 845557); Dwyer Hill Road N of Burritts Rapids, 45.0267, -75.8582, 7 November 2009, P.M. Catling (DAO 845561); Flood Road, 45.0352, -75.8134, 7 November 2009, P.M. Catling s.n. (DAO 845575); Flood Road 2, Marlborough Forest, 45.0406, -75.8100, 7 November 2009, P.M. Catling s.n. (DAO 845574); Mill Street S of Merrickville, 44.8882, -75.8198, 12 November 2009, P.M. Catling s.n. (DAO 845580); N of North Augustusta, 44.7809, -75.7571, 12 November 2009, P.M. Catling s.n. (DAO 845579); Asselstine alvar, 44.2612, -76.7104, 22 November 2009, P.M. Catling s.n. (DAO 844681); Asselstine alvar, 44.2612, -76.7104, 22 November 2009, P.M. Catling s.n. (DAO 844680); Grant Side Road [15 km SE of Arnprior], 45.3608, -76.1794, 22 November 2009, P.M. Catling s.n. (DAO 843931); Dwyer and O'Neil Roads [20 km NNE of Smiths Falls], 45.0792, -75.9058, 28 November 2009, P.M. Catling s.n. (DAO 843935); Franktown, 45.0096, -76.0529, 28 November 2009, P.M. Catling s.n. (DAO 843936).

Sight record. CANADA. Ontario: O'Neil $\operatorname{Road}[13.5 \mathrm{~km}$ NW of BurriRapids], 45.08, -75.904, 28 2009, P.M. Catling.

\section{Sporobolus vaginiflorus var. vaginiflorus}

CANADA. Ontario: Thurlow Twp., Belleville, 44.2567, -77.3697, September 1871, J. Macoun s.n. (QK 55478); Britannia, 45.3715, -75.8038, September 1919, Rolland-Germain 8023 (DAO 623680); Britannia, 45.3735, -75.7975, September 1919, Fr. Rolland-Germain 8023 (CAN 355410); Kinburn, 45.354, -76.1901, 3 September 1943, W.G. Dore \& H. Groh 5072 (DAO 221046); Shirleys Bay, 45.3658, -75.8828, 13 September 1952, W.G. Dore \& D. Erskine 14094 (DAO 221048); Burnt Lands [5 km NE of Almonte], 45.2562, -76.1480, 22 November 1953, C. Frankton 1489 (DAO
221045); Westboro, Ottawa, 45.382, -75.7767, 28 August 1955, W.G. \& K.M. Dore 16164 (DAO 221103); Huntley Twp., $5 \mathrm{~km}$ ENE of Almonte, 45.225, -76.195, 7 June 1969, R. Beschell \& E. Greenwood 17202 (QK 91964); Tyendinaga Twp., Highway 401, W of Salmon River, 44.2287, -77.1662, 1 May 1970, R. Beschel 100 (QK 97216); Burnt Lands, 45.2562, -76.1480, 8 September 1974, P.M. Catling and S.M. McKay (DAO 131201); Huntley Twp., Burnt Lands NE of Almonte, 45.25, -76.1000, 8 September 1974, P.M. Catling \& S.M. McKay (CAN 394675); Burnt Lands, 3 miles NE of Almonte, N side of Hwy. 44, 45.2666, -76.15, 26 August 1978, David White 1234 (CAN 433138); Ottawa River, Shirleys Bay, $1 \mathrm{~km}$ NW of Innis Point, 45.3833, -75.8833, 28 August 1980, D.F. Brunton 2573 (CAN 455766); Almonte alvar, 45.2562, -76.148, 28 August 1983, R. Hainault 8361 (DAO 839501); Braeside, $3.5 \mathrm{~km} \mathrm{NW}$ of, 45.4763, -76.4485, 11 October 1983, S.J. Darbyshire 2131 (DAO 383315); Malloy Bay, Ottawa River [7.5 km NE of Westmeath], 45.8666, -76.7943, 6 November 1983, S.J. Darbyshire 2150 (DAO 396266); Westmeath Twp., North Front D, Ottawa River, Malloy Bay, 45.8666, -76.7944, 6 November 1983, S.J. Darbyshire 2150 (CAN 543627); $3 \mathrm{~km} \mathrm{~W}$ of Panmure, 45.3166 , -76.2333, 25 September 1985, D.F. Brunton 6585 (DAO 452153); Sand Point, $1.5 \mathrm{~km} \mathrm{SW,} \mathrm{45.7833,} \mathrm{-76.9166,} 26$ August 1987, D.F. Brunton 7594 (DAO 572863); Clay Bank alvar, 45.3741, -76.4071, 6 October 1987, S.J. Darbyshire $3642 a$ (DAO 575687); SE part of MacNab Twp., along border with Lanark County, 45.3833, -76.3833, 6 October 1988, S.J. Darbyshire 3642A (CAN 529796); Mohawk Cemetery, 44.1813, -77.0816, 19 September 1995, M.J. Oldham \& W.J. Bakowsky 18246 (DAO 688984); $6 \mathrm{~km}$ NNW of Lonsdale, 44.299, -77.1904, 19 September 1995, M.J. Oldham \& W.J. Bakowsky 18230 (DAO 701807); Ramsay alvar (Thyrea site), 45.2639, -76.1935, 14 October 2009, G. Mitrow, P.M. Catling, I. McFetridge, \& S. Chan (DAO 844215); Ramsay alvar (Thyrea site), 45.2639, -76.1935, 14 October 2009, G. Mitrow, P.M. Catling, I. McFetridge \& S. Chan s.n. (DAO 844217); Ramsay alvar (Thyrea site), 45.2639, -76.1935, 14 October 2009, G. Mitrow, P.M. Catling, I. McFetridge \& S. Chan s.n. (DAO 844219); Ramsay alvar (Thyrea site), 45.2639, -76.1935, 14 October 2009, G. Mitrow, P.M. Catling, I. McFetridge \& S. Chan s.n. (DAO 844218); Ramsay alvar (Thyrea site), 45.2639, -76.1935, 14 October 2009, G. Mitrow, P.M. Catling, I. McFetridge \& S. Chan s.n. (DAO 844214); Ramsay alvar (Thyrea site), 45.2639, -76.1935, 14 October 2009, G. Mitrow, P.M. Catling, I. McFetridge \& S. Chan s.n. (DAO 844212); Ramsay alvar (Thyrea site), 45.2639, -76.1935, 14 October 2009, G. Mitrow, P.M. Catling, I. McFetridge \& S. Chan s.n. (DAO 844209); Ramsay alvar (Thyrea site), 45.2639, -76.1935, 14 October 2009, G. Mitrow, P.M. Catling, I. McFetridge \& S. Chan s.n. (DAO 844208); Ramsay alvar (Thyrea site), 45.2639, -76.1935, 14 October 2009, G. Mitrow, P.M. Catling, I. McFetridge \& S. Chan s.n. (DAO 844213); Ramsay alvar (Thyrea site), 45.2639, -76.1935, 14 October 2009, G. Mitrow, P.M. Catling, I. McFetridge \& S. Chan s.n. (DAO 844216); Ramsay alvar (Thyrea site), 45.2639, -76.1935, 14 October 2009, G. Mitrow, P.M. Catling, I. McFetridge \& S. Chan s.n. (DAO 844210); Ramsay alvar (Thyrea site), 45.2639, -76.1935, 14 October 2009, G. Mitrow, P.M. Catling, I. McFetridge \& S. Chan s.n. (DAO 844211); Ramsay alvar (Big Prairie), 45.2738, -76.1934, 
14 October 2009, G. Mitrow, P.M. Catling, I. McFetridge \& S. Chan s.n. (DAO 844228); Ramsay alvar (Big Prairie), 45.2738, -76.1934, 14 October 2009, G. Mitrow, P.M. Catling, I. McFetridge \& S. Chan s.n. (DAO 844226); Ramsay alvar (Big Prairie), 45.2738, -76.1934, 14 October 2009, G. Mitrow, P.M. Catling, I. McFetridge \& S. Chan s.n. (DAO 844231); Ramsay alvar (Big Prairie), 45.2738, -76.1934, 14 October 2009, G. Mitrow, P.M. Catling, I. McFetridge \& S. Chan s.n. (DAO 844230); Ramsay alvar (Big Prairie), 45.2738, -76.1934, 14 October 2009, G. Mitrow, P.M. Catling, I. McFetridge \& S. Chan s.n. (DAO 844227); extension of Ramsay Concession 12, 45.2652, -76.189, 14 October 2009, G. Mitrow, P.M. Catling, I. McFetridge \& S. Chan s.n. (DAO 844220); extension of Ramsay Concession 12, 45.2652, -76.189, 14 October 2009, G. Mitrow, P.M. Catling, I. McFetridge \& S. Chan s.n. (DAO 844224); extension of Ramsay Concession 12, 45.2652, -76.189, 14 October 2009, G. Mitrow, P.M. Catling, I. McFetridge \& S. Chan s.n. (DAO 844225); extension of Ramsay Concession 12, 45.2652, -76.189, 14 October 2009, G. Mitrow, P.M. Catling, I. McFetridge \& S. Chan s.n. (DAO 844223); Odessa alvar, 44.2663, -76.7104, 18 October 2009, P.M. Catling \& B. Kostiuk s.n.
(DAO 844864); Odessa alvar, 44.2663, -76.7104, 18 October 2009, P.M. Catling \& B. Kostiuk s.n. (DAO 843818); Clay Bank alvar, 9th Concession at Barrie Road [Pakenham Twp., Ottawa], 45.3739, -76.4072, 25 October 2009, P.M. Catling \& B. Kostiuk s.n. (DAO 843920); Department of National Defence (DND) alvar, Burnt Lands Provincial Park, 45.255, -76.1484, 25 October 2009, P.M. Catling \& B. Kostiuk s.n. (DAO 845578); Department of Natoinal Defence (DND) alvar, Burnt Lands Provincial Park, 45.255, -76.1484, 25 October 2009, P.M. Catling \& B. Kostiuk s.n. (DAO 845577); Burnt Lands E of Golden Line, 45.2488, -76.1417, October 2009, P.M. Catling (DAO 844688); Braeside, 45.4789, -76.4477, October 2009, P.M. Catling \& B. Kostiuk s.n. (DAO 844687); Asselstine alvar, 44.2612, -76.7104, 22 November 2009, P.M. Catling s.n. (DAO 844682); Chartrand Island, Shirleys Bay, Ottawa River, 45.3848, -75.8973, s.d., J.M. Gillet s.n. (DAO 220864); Beattie [Beatty] Point, 45.3658, -75.8828, s.d., H. Groh s.n. (DAO 221041); Shirleys Bay, 45.3658, -75.8828, s.d., W.G. Dore s.n. (DAO 221043); Shirleys Bay, s.d., 45.3658, -75.8828, W.G. Dore s.n. (DAO 221044). 
TABLE 1. Co-occurrence data for 200 plants at each of 64 sites (4 river shores, 23 alvars, and 22 roadsides). The data include the percentage of 200 plants examined of each taxon at each site and include 32 occurrences of Sporobolus vaginiflorus var. inaequalis, 18 of Sporobolus vaginiflorus var. ozarkanus, and 8 of Sporobolus vaginiflorus var. vaginiflorus.

\begin{tabular}{|c|c|c|c|c|c|}
\hline & \multirow[b]{2}{*}{ Latitude } & \multirow[b]{2}{*}{ Longitude } & \multicolumn{3}{|c|}{ Sporobolus vaginiflorus } \\
\hline & & & $\begin{array}{c}\% \text { var. } \\
\text { inaequalis }\end{array}$ & $\begin{array}{c}\% \text { var. } \\
\text { ozarkanus }\end{array}$ & $\begin{array}{c}\% \text { var. } \\
\text { vaginiflorus }\end{array}$ \\
\hline \multicolumn{6}{|l|}{ Roadsides, railways, gravel pits (22) } \\
\hline & 44.56880 & -77.11654 & 100 & 0 & 0 \\
\hline & 44.64820 & -77.12171 & 100 & 0 & 0 \\
\hline & 44.75440 & -76.85267 & 100 & 0 & 0 \\
\hline & 44.85436 & -76.44313 & 100 & 0 & 0 \\
\hline & 44.90908 & -76.26596 & 100 & 0 & 0 \\
\hline & 45.08001 & -75.90396 & 80 & 20 & 0 \\
\hline & 45.14285 & -77.26627 & 100 & 0 & 0 \\
\hline & 45.18773 & -76.99974 & 100 & 0 & 0 \\
\hline & 45.21290 & -77.44009 & 100 & 0 & 0 \\
\hline & 45.22298 & -76.95741 & 100 & 0 & 0 \\
\hline & 45.26049 & -75.97174 & 100 & 0 & 0 \\
\hline & 45.29506 & -77.44725 & 100 & 0 & 0 \\
\hline & 45.31976 & -75.73569 & 100 & 0 & 0 \\
\hline & 45.35482 & -75.46777 & 100 & 0 & 0 \\
\hline & 45.35680 & -75.73687 & 100 & 0 & 0 \\
\hline & 45.36068 & -75.88069 & 100 & 0 & 0 \\
\hline & 45.36397 & -75.87257 & 100 & 0 & 0 \\
\hline & 45.38600 & -75.34857 & 100 & 0 & 0 \\
\hline & 45.43386 & -77.31621 & 100 & 0 & 0 \\
\hline & 45.44675 & -76.55659 & 100 & 0 & 0 \\
\hline & 45.49621 & -76.66999 & 100 & 0 & 0 \\
\hline & 45.59506 & -74.61571 & 100 & 0 & 0 \\
\hline \multicolumn{6}{|l|}{ Alvars (23) } \\
\hline \multicolumn{6}{|l|}{ Smiths Falls Plain (15) } \\
\hline & 44.78088 & -75.75705 & 0 & 100 & 0 \\
\hline & 44.88815 & -75.81976 & 0 & 100 & 0 \\
\hline & 45.00958 & -76.05291 & 0 & 100 & 0 \\
\hline & 45.02667 & -75.85820 & 0 & 100 & 0 \\
\hline & 45.03521 & -75.81342 & 0 & 100 & 0 \\
\hline & 45.04058 & -75.80996 & 0 & 100 & 0 \\
\hline & 45.07749 & -75.81175 & 43 & 57 & 0 \\
\hline & 45.07924 & -75.90578 & 0 & 100 & 0 \\
\hline & 45.24879 & -76.14172 & 0 & 0 & 100 \\
\hline & 45.25621 & -76.14804 & 0 & 0 & 100 \\
\hline & 45.26390 & -76.19350 & 0 & 0 & 100 \\
\hline & 45.27380 & -76.19340 & 0 & 0 & 100 \\
\hline & 45.36080 & -76.17938 & 0 & 100 & 0 \\
\hline & 45.37413 & -76.40706 & 0 & 0 & 100 \\
\hline & 45.47886 & -76.44771 & 0 & 0 & 100 \\
\hline \multicolumn{6}{|l|}{ Napanee Plain (8) } \\
\hline & 44.15751 & -77.29488 & 0 & 100 & 0 \\
\hline & 44.16769 & -77.30115 & 0 & 100 & 0 \\
\hline & 44.17756 & -77.11947 & 45 & 55 & 0 \\
\hline & 44.18126 & -77.08159 & 0 & 100 & 0 \\
\hline & 44.25310 & -77.12210 & 39 & 61 & 0 \\
\hline & 44.26120 & -76.71037 & 55 & 30 & 15 \\
\hline & 44.26626 & -76.71037 & 38 & 40 & 22 \\
\hline & 44.33420 & -76.79290 & 53 & 47 & 0 \\
\hline \multicolumn{6}{|l|}{ Shore of the Ottawa River (4) } \\
\hline & 45.36583 & -75.88284 & 100 & 0 & 0 \\
\hline & 45.38639 & -75.89383 & 100 & 0 & 0 \\
\hline & 45.39148 & -75.89811 & 100 & 0 & 0 \\
\hline & 45.51126 & -76.22361 & 100 & 0 & 0 \\
\hline
\end{tabular}

